\title{
Armazenamento de sementes de Alfavaca-cravo (Ocimum gratissimum L.)
}

MARTINS, J.R. ${ }^{*}$; NEVES, C.L.P.1; PEREIRA, W.V.S.'; TONETTI, O.A.O.1; ALVARENGA, A.A. ${ }^{2}$

1Universidade Federal de Lavras (UFLA), Departamento de Ciências Florestais, Campus Universitário, Caixa Postal: 3037, CEP: 37.200-000, Lavras-Brasil *joefersonreis@yahoo.com.br. ${ }^{2}$ Universidade Federal de Lavras (UFLA), Departamento de Biologia, Campus Universitário, Caixa Postal: 3037, CEP: 37.200-000, Lavras-Brasil

\begin{abstract}
RESUMO: A espécie Ocimum gratissimum L. é um subarbusto aromático, pertencente à família Lamiaceae, considerado como importante produtor de óleo essencial e largamente utilizado na medicina popular. Devido à escassez de informações sobre a conservação das sementes dessa espécie o presente estudo objetivou analisar a longevidade das sementes submetidas a diferentes condições de armazenamento, por 12 meses, através de avaliações do potencial germinativo e da capacidade de formação de plântulas normais. As sementes foram armazenadas em três tipos de embalagens: sacos de papel, sacos de papel aluminizado, e frascos de vidro, e nas seguintes condições ambientais: balcão de laboratório, geladeira, e câmara fria. Aos 6, 8, 10 e 12 meses de armazenamento foram avaliados os seguintes parâmetros: umidade, porcentagem de germinação, índice de velocidade de germinação e porcentagem de plântulas normais. Durante o período de armazenamento a manutenção da umidade nas sementes foi mantida, bem como a porcentagem de germinação e de plântulas normais em todos os tratamentos avaliados. As sementes armazenadas apresentaram maiores valores de IVG quando comparadas com as recém-colhidas.
\end{abstract}

Palavras-chave: Germinação, qualidade de sementes, longevidade, plântulas normais, planta medicinal.

ABSTRACT: Storage of Clove Basil (Ocimum gratissimum L.) seeds. The Ocimum gratissimum L. species is an aromatic subshrub that belongs to the Lamiaceae family and is considered as an important producer of essential oil, widely used in folk medicine. Because of the lack of information about the conservation of $O$. gratissimum seeds, we aimed to evaluate the longevity of the seeds on different storage conditions for 12 months through the germination and seedling formation percentage in this study. The seeds were stored in three types of packages: paper bags (permeable), aluminized paper bags (semipermeable) and glass flasks (impermeable), in the settings of laboratory bench, refrigerator and cold room over the 12 month period. The germination tests were performed on the 6th, 8th, 10th and 12th month, when moisture, germinability, germination velocity index and percentage of normal seedlings were evaluated. During the storage period, moisture, germinability and percentage of normal seedling were kept in each storage condition. The stored seeds showed increased values of GSI compared to the newly- harvested ones.

Keywords: Germination, seed quality, longevity, seedlings, medicinal plant.

\section{INTRODUÇÃO}

Ocimum gratissimum L. (Lamiaceae), conhecido popularmente como Alfavaca-cravo, é um subarbusto lenhoso, ereto, com até $1,0 \mathrm{~m}$ de altura, originário da Ásia e África e subespontâneo em todo território brasileiro. Essa espécie apresenta propriedades medicinais importantes e por isso é largamente utilizada no Brasil como medicamento complementar à alopatia. O óleo essencial presente nas suas folhas tem ação inibitória sobre organismos altamente patogênicos, como Staphylococcus aureus, Bacillus spp, Pseudomonas aeruginosae e Leishmania amazonensis (Ueda-Nakamura et al., 2006; Matasyoh et al., 2007), além disso, tem sido considerado como uma alternativa natural aos inseticidas sintéticos no combate às pragas infestantes de grãos armazenados (Ogendo et al., 2008).

Devido às características de interesse 
medicinal, pesquisas sobre os métodos de propagação que proporcionem melhorias na germinação, e outras sobre a produtividade do óleo essencial têm sido encontradas na literatura (Fiallo et al., 1996; Martins et al., 2008a; Martins et al., 2008b). No entanto, as informações a respeito dos métodos de conservação das sementes de $O$. gratissimum ainda são escassas. Nesse sentido, o presente estudo buscou analisar a longevidade das sementes armazenadas por 12 meses em diferentes tipos de embalagem, acondicionadas em ambientes distintos, avaliando o potencial de germinação e a capacidade de formação de plântulas normais.

\section{MATERIAL E MÉTODO}

As sementes de alfavaca-cravo foram coletadas de plantas adultas do Horto de Plantas Medicinais do Campus da Universidade Federal de Lavras (UFLA), em maio de 2005, das quais foram feitas exsicatas para identificação taxonômica e preservação no Herbário ESAL, do Departamento de Biologia da UFLA, sob o número de registro 20.0037.

Os experimentos foram realizados no Departamento de Biologia da Universidade Federal de Lavras a partir de sementes beneficiadas por meio de soprador pneumático.

A umidade das sementes foi determinada pelo método da estufa a $105 \pm 2^{\circ} \mathrm{C}$, por 24 horas, em sementes recém-colhidas, bem como, nas sementes submetidas ao armazenamento em cada período de avaliação, utilizando-se cinco replicas de dois gramas cada.

O experimento de armazenamento foi instalado com sementes previamente tratadas com o fungicida Captan 2\% (Brasil, 1992). Utilizouse o delineamento inteiramente casualizado, com quatro repetições de dois gramas cada, em esquema fatorial $4 \times 3 \times 3$, sendo quatro períodos de avaliações: 6, 8, 10 e 12 meses; três tipos de embalagens: sacos de papel kraft, sacos de papel aluminizado, e frascos de vidro transparente; e três ambientes de armazenamento: sobre balcão de laboratório, câmara fria com $5 \pm 3^{\circ} \mathrm{C}$ e $45 \%$ de umidade relativa (UR), e geladeira a $10 \pm 3^{\circ} \mathrm{C}$ e $50 \%$ (UR). As variações no ambiente de laboratório acompanharam as mudanças climáticas da cidade de Lavras no período experimental de maio de 2005 a maio 2006. Nos outros ambientes de armazenamento, os dados de temperatura foram monitorados com termômetro de máxima/mínima e a umidade, com psicrômetro e termo-higrômetro digital.

A avaliação da longevidade das sementes em cada período de armazenamento foi realizada através de testes de germinação em caixas plásticas gerbox, forradas com folha dupla de papel germtest e umedecidas com água destilada na quantidade correspondente a 2,5 vezes o peso do substrato, acondicionadas em câmara BOD, sob temperatura alternada de 20 e $30^{\circ} \mathrm{C}$, e fotoperíodo de 12 horas. Cada tratamento foi avaliado a partir de quatro replicas de 100 sementes. A contagem de plantas germinadas, considerando a protrusão radicular, foi diária durante o período de 14 dias.

A partir dos dados de contagem de germinação foi obtido o índice de velocidade de germinação (IVG), mensurado pela equação proposta por Maguire (1962). Ao final de 14 dias, o número de plântulas normais foi contabilizado considerado as

TABELA 1. Variação percentual da umidade das sementes de O. gratissimum ao armazenamento por 12 meses, em diferentes embalagens e condições ambientais.

\begin{tabular}{|c|c|c|c|c|c|}
\hline \multirow{2}{*}{ Ambiente } & \multirow{2}{*}{ Embalagem } & \multicolumn{4}{|c|}{ Tempo de armazenamento } \\
\hline & & 6 meses & 8 meses & 10 meses & 12 meses \\
\hline Balcão & Vidro & $8,33 \pm 0,009$ & $8,28 \pm 0,007$ & $8,28 \pm 0,009$ & $8,49 \pm 0,017$ \\
\hline Balcão & P. Alum. & $8,28 \pm 0,013$ & $8,46 \pm 0,007$ & $8,28 \pm 0,007$ & $8,53 \pm 0,014$ \\
\hline Balcão & Papel & $8,10 \pm 0,011$ & $8,21 \pm 0,010$ & $8,38 \pm 0,016$ & $8,21 \pm 0,008$ \\
\hline Geladeira & Vidro & $8,23 \pm 0,008$ & $8,28 \pm 0,016$ & $8,28 \pm 0,009$ & $8,05 \pm 0,015$ \\
\hline Geladeira & P. Alum. & $8,11 \pm 0,011$ & $8,28 \pm 0,009$ & $8,06 \pm 0,013$ & $8,10 \pm 0,021$ \\
\hline Geladeira & Papel & $7,95 \pm 0,014$ & $8,00 \pm 0,017$ & $8,20 \pm 0,011$ & $8,00 \pm 0,018$ \\
\hline Câm. Fria & Vidro & $8,31 \pm 0,009$ & $8,32 \pm 0,008$ & $8,31 \pm 0,006$ & $8,34 \pm 0,010$ \\
\hline Câm. Fria & P. Alum. & $8,43 \pm 0,007$ & $8,00 \pm 0,010$ & $8,49 \pm 0,020$ & $8,41 \pm 0,019$ \\
\hline Câm. Fria & Papel & $8,32 \pm 0,014$ & $8,05 \pm 0,009$ & $8,54 \pm 0,017$ & $8,52 \pm 0,013$ \\
\hline $\mathrm{N}$ & & 5 & 5 & 5 & 5 \\
\hline
\end{tabular}

* Legenda: Vidro - frasco de vidro, P. Alum. - saco de papel aluminizado, Papel - saco de papel, Câm. Fria - câmara fria. 
seguintes características morfológicas: presença de raiz primária, caulículo ereto e cotilédones abertos e verdes.

Os dados foram submetidos à ANOVA e as médias comparadas utilizando o teste Tukey, através do programa estatístico SISVAR (Ferreira, 1999). Os dados de germinação foram previamente transformados em arcsen (X/100).0,5.

\section{RESULTADO E DISCUSSÃO}

As sementes de $O$. gratissimum recém colhidas e beneficiadas apresentaram umidade de $8,28 \%$, este teor de água não variou significantemente ao longo dos 12 meses de armazenamento, mesmo nas sementes mantidas em embalagem permeável sob condição ambiental de laboratório, com umidade variável (Tabela 1 e Figura 1).

A baixa variação na umidade das sementes O. gratissimum durante armazenamento pode estar relacionada aos elevados teores de lipídios e amido, substâncias de reserva pouco solúveis (Martins, 2006). De acordo com Marcos Filho (2005), a umidade é um dos fatores que mais afetam a atividade metabólica das sementes e, por isso, pode determinar a manutenção da viabilidade embrionária e da qualidade das sementes. Assim, a baixa higroscopicidade das sementes de $O$. gratissimum pode estar associada ao mecanismo de conservação da qualidade e longevidade das sementes, por meio da inibição de amplas variações de umidade.

A longevidade em sementes ortodoxas é amplamente relatada na literatura (Roberts,
1973; Davide et al. 2003; Carvalho et al. 2006). Nagel \& Böner (2010) verificaram a longevidade em sementes ortodoxas de 18 espécies mantidas sob condições ambientais $\left(20,3^{\circ} \mathrm{C}\right.$ e $50,5 \%$ UR $)$ por até 23 anos. Entretanto, estudos sobre os mecanismos que asseguram a preservação da viabilidade embrionária e a qualidade das sementes em ambientes com ampla variação de temperatura e umidade são escassos.

A porcentagem de germinação das sementes recém-colhidas foi de $52,5 \%$ e mantevese semelhante durante todo o período de armazenamento em todas as condições avaliadas (Tabela 2). Resultado semelhante foi descrito por Fiallo et al. (1996), o qual verificou que, mesmo sob adições exógenas de ácido giberélico, a germinação dessa espécie não excedeu a $62 \%$. A baixa porcentagem de germinação em 0 . gratissimum tem sido relacionada à grande produção de sementes vazias, cerca de 52\% (Martins et al. 2008a).

A manutenção da longevidade das sementes foi mantida por 12 meses, mesmo quando armazenadas em embalagem permeável sob condição ambiental de laboratório. Este comportamento, possivelmente está relacionado à baixa variação de umidade da semente ao longo do armazenamento, o que teria impedido o aumento da taxa de respiração e evitado reações degenerativas.

As sementes submetidas ao armazenamento germinaram mais rapidamente que as sementes recém-colhidas, IVG 14,69 (Tabela 3). O aumento da velocidade de germinação foi maior para as sementes armazenadas em baixas temperaturas, geladeira e câmara fria, independentemente do

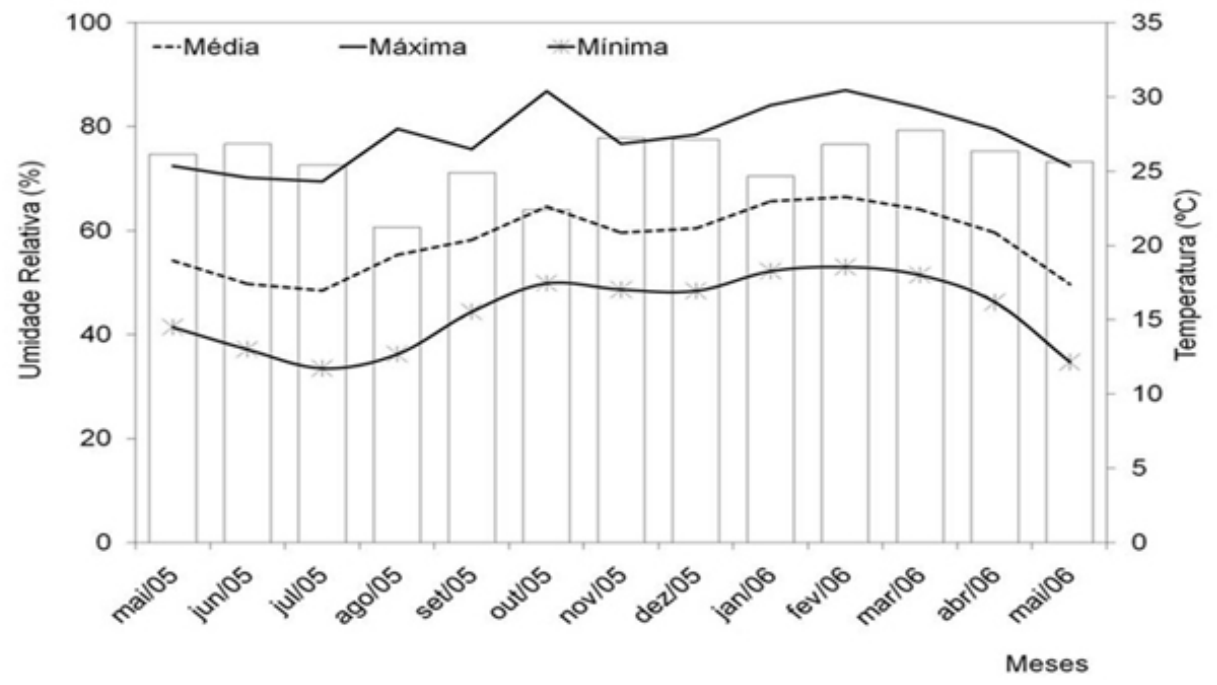

FIGURA 1. Valores médios de umidade relativa do ar e temperaturas ao longo do período de armazenamento das sementes de O. gratissimum (maio de 2005 a maio de 2006). Fonte: Estação Meteorológica da Universidade Federal de Lavras, Lavras-MG. Legenda: UR\%= Percentual de Umidade Relativa do Ar; Média=Valores médios de temperatura; Mínima= Temperatura Mínima; Máxima= Temperatura máxima. 
TABELA 2. Percentual de germinação de sementes de O. gratissimum armazenadas sob diferentes condições ambientais e embalagens.

\begin{tabular}{cccccc}
\hline & & \multicolumn{4}{c}{ Tempo de armazenamento } \\
\cline { 3 - 6 } Ambiente & Embalagem & 6 meses & 8 meses & 10 meses & 12 meses \\
\hline Balcão & Vidro & $56,00 \mathrm{aA} *$ & $56,75 \mathrm{aA}$ & $48,00 \mathrm{bA}$ & $50,25 \mathrm{bB}$ \\
Balcão & P. Alum & $51,75 \mathrm{aA}$ & $49,00 \mathrm{aA}$ & $48,00 \mathrm{aA}$ & $50,00 \mathrm{aB}$ \\
Balcão & Papel & $53,50 \mathrm{aA}$ & $52,00 \mathrm{aA}$ & $54,50 \mathrm{aA}$ & $59,00 \mathrm{aA}$ \\
\hline Geladeira & Vidro & $49,75 \mathrm{bA}$ & $56,50 \mathrm{aA}$ & $48,00 \mathrm{bA}$ & $54,00 \mathrm{aA}$ \\
Geladeira & P. Alum. & $48,50 \mathrm{aA}$ & $52,00 \mathrm{aA}$ & $43,75 \mathrm{bB}$ & $53,75 \mathrm{aA}$ \\
Geladeira & Papel & $53,00 \mathrm{aA}$ & $54,00 \mathrm{aA}$ & $54,25 \mathrm{aA}$ & $53,00 \mathrm{aA}$ \\
\hline Câm. Fria & Vidro & $51,50 \mathrm{aA}$ & $53,25 \mathrm{aA}$ & $44,75 \mathrm{aA}$ & $49,00 \mathrm{aA}$ \\
Câm. Fria & P. Alum. & $43,50 \mathrm{bB}$ & $50,25 \mathrm{bA}$ & $56,75 \mathrm{aB}$ & $56,25 \mathrm{aA}$ \\
Câm. Fria & Papel & $53,75 \mathrm{bA}$ & $51,50 \mathrm{bA}$ & $51,25 \mathrm{bB}$ & $60,00 \mathrm{aA}$ \\
\hline
\end{tabular}

${ }^{*}$ As letras minúsculas comparam os tempos, na linha $(P<0,05)$. As letras maiúsculas comparam embalagens, na coluna, para cada ambiente $(P<0,05)$. Legenda: Vidro =frasco de vidro; $P$. Alum = saco de papel aluminizado; Papel= saco de papel; Câm. Fria = câmara fria.

TABELA 3. Índice de velocidade de germinação (IVG) de sementes de O. gratissimum armazenadas sob diferentes condições ambientais e embalagens.

\begin{tabular}{cccccc}
\hline & & \multicolumn{4}{c}{ Tempo de armazenamento } \\
\cline { 3 - 5 } Ambiente & Embalagem & $\mathbf{6}$ meses & $\mathbf{8}$ meses & $\mathbf{1 0}$ meses & $\mathbf{1 2}$ meses \\
\hline Balcão & Vidro & $17,30 \mathrm{aA}^{*}$ & $17,62 \mathrm{aA}$ & $15,65 \mathrm{aA}$ & $16,36 \mathrm{aA}$ \\
Balcão & P. Alum & $15,50 \mathrm{aA}$ & $13,58 \mathrm{aB}$ & $15,88 \mathrm{aA}$ & $15,82 \mathrm{aA}$ \\
Balcão & Papel & $16,24 \mathrm{aA}$ & $16,15 \mathrm{aA}$ & $17,35 \mathrm{aA}$ & $18,18 \mathrm{aA}$ \\
\hline Geladeira & Vidro & $15,50 \mathrm{cA}$ & $18,68 \mathrm{bA}$ & $17,60 \mathrm{bA}$ & $23,14 \mathrm{aA}$ \\
Geladeira & P. Alum. & $15,50 \mathrm{cA}$ & $18,68 \mathrm{bA}$ & $17,77 \mathrm{bA}$ & $24,31 \mathrm{aA}$ \\
Geladeira & Papel & $16,45 \mathrm{cA}$ & $20,45 \mathrm{bA}$ & $20,02 \mathrm{bA}$ & $23,59 \mathrm{aA}$ \\
\hline Câm. Fria & Vidro & $15,36 \mathrm{cA}$ & $18,13 \mathrm{bA}$ & $21,54 \mathrm{aB}$ & $21,50 \mathrm{aB}$ \\
Câm. Fria & P. Alum. & $13,54 \mathrm{cA}$ & $17,98 \mathrm{bA}$ & $27,38 \mathrm{aA}$ & $25,28 \mathrm{aA}$ \\
Câm. Fria & Papel & $16,49 \mathrm{bA}$ & $16,91 \mathrm{bA}$ & $24,56 \mathrm{aA}$ & $25,27 \mathrm{aA}$ \\
\hline
\end{tabular}

*Letras minúsculas comparam os tempos, na linha $(P<0,05)$. As letras maiúsculas comparam embalagens, na coluna, para cada ambiente $(P<0,05)$. Legenda: Vidro $=$ frasco de vidro; $\mathbf{P}$. Alum. $=$ saco de papel aluminizado; Papel = saco de papel; Câm. Fria = câmara fria .

tipo de embalagem (Tabela 3). Scalon et al. (2006) verificaram que o armazenamento sob refrigeração proporcionou melhora do percentual e velocidade de germinação de sementes de Jacarandá cuspidifolia. De acordo com Fiallo et al. (1996), o aumento na velocidade de germinação, com o armazenamento, constitui uma defesa de $O$. gratissimum para perpetuar-se, ao evitar ou minimizar a competição com outras espécies no uso de recursos naturais limitantes ao desenvolvimento das plântulas, tais como: luz, água e nutrientes.

Aformação de plântulas a partir de sementes recém-colhidas foi de $47 \%$. Após o armazenamento, assim como verificado na germinação inicial, houve manutenção do percentual de formação de plântulas normais, revelando a preservação da qualidade fisiológica da semente durante o período do estudo em todos os tratamentos (Tabela 4). A formação de plântula normal é primordial para a produção de mudas saudáveis e está diretamente associado com o poder germinativo e a manutenção da qualidade fisiológica da semente (Tresena et al. 2009).

Os resultados obtidos no presente estudo demonstraram que as sementes de 0 . gratissimum se mantiveram viáveis durante 12 meses sob as diferentes condições de armazenamento avaliadas. A baixa variação da umidade das sementes ao longo do período de armazenamento pode estar associada à manutenção da qualidade fisiológica das sementes, constatada pela maior velocidade 
TABELA 4. Percentual de plântulas normais de sementes germinadas de O. gratissimum armazenadas sob diferentes condições ambientais e embalagens.

\begin{tabular}{cccccc}
\hline & & \multicolumn{4}{c}{ Tempo de armazenamento } \\
\cline { 3 - 6 } Ambiente & Embalagem & $\mathbf{6}$ meses & $\mathbf{8}$ meses & $\mathbf{1 0}$ meses & $\mathbf{1 2}$ meses \\
\hline Balcão & Vidro & $54,25 \mathrm{aA}$ & $53,50 \mathrm{aA}$ & $47,00 \mathrm{bA}$ & $49,00 \mathrm{bB}$ \\
Balcão & P. Alum & $48,25 \mathrm{aA}$ & $46,25 \mathrm{aA}$ & $46,75 \mathrm{aA}$ & $49,50 \mathrm{aB}$ \\
Balcão & Papel & $51,75 \mathrm{aA}$ & $51,00 \mathrm{aA}$ & $53,25 \mathrm{aA}$ & $58,50 \mathrm{aA}$ \\
\hline Geladeira & Vidro & $48,00 \mathrm{bA}$ & $54,75 \mathrm{aA}$ & $46,75 \mathrm{bA}$ & $53,25 \mathrm{aA}$ \\
Geladeira & P. Alum. & $47,00 \mathrm{aA}$ & $51,25 \mathrm{aA}$ & $40,00 \mathrm{bB}$ & $52,50 \mathrm{aA}$ \\
Geladeira & Papel & $51,00 \mathrm{aA}$ & $53,50 \mathrm{aA}$ & $53,50 \mathrm{aA}$ & $52,25 \mathrm{aA}$ \\
\hline Câm. Fria & Vidro & $47,75 \mathrm{aA}$ & $51,75 \mathrm{aA}$ & $44,25 \mathrm{aB}$ & $48,25 \mathrm{aB}$ \\
Câm. Fria & P. Alum. & $40,25 \mathrm{bB}$ & $48,50 \mathrm{aA}$ & $56,50 \mathrm{aA}$ & $54,50 \mathrm{aA}$ \\
Câm. Fria & Papel & $52,50 \mathrm{bA}$ & $50,50 \mathrm{bA}$ & $50,75 \mathrm{bA}$ & $59,25 \mathrm{aA}$ \\
\hline
\end{tabular}

*As letras minúsculas comparam os tempos, na linha $(P<0,05)$. As letras maiúsculas comparam embalagens, na coluna, para cada ambiente $(\mathrm{P}<0,05)$. Legenda: Vidro = frasco de vidro; $\mathrm{P}$. Alum. $=$ saco de papel aluminizado; Papel $=$ saco de papel; Câm. Fria $=$ câmara fria.

de germinação e preservação da porcentagem de formação de plântulas normais no final do período avaliado.

\section{AGRADECIMENTO}

Ao Prof. Dr. Renato Mendes Guimarães e ao Prof. Dr. João Almir Oliveira do Departamento de Agricultura da UFLA pelas valiosas sugestões e à FAPEMIG, pelo apoio financeiro.

\section{REFERÊNCIA}

BRASIL. Regras para análise de sementes. Brasília: Departament, 1992. 365p.

CARVALHO, L.R. et al. Classificação de sementes florestais quanto ao comportamento no armazenamento. Revista Brasileira de Sementes, v. 28, n. 2, p.15-25, 2006.

DAVIDE, A.C. et al. Classificação fisiológica de sementes de espécies florestais pertencentes à família lauraceae quanto à capacidade de armazenamento. Cerne, Lavras, v.9, n.1 p.29-35, 2003.

FERREIRA, D.F. SISVAR 4. 3-Sistema de analises estatísticas. Lavras: UFLA, 1999.

FIALLO, V.R.F. et al. Acerca de la propagación de Ocimum gratissimum L. Revista Cubana Planta Medica, v.1, n.1, p.3-7, 1996.

MAGUIRE, J.D. Speed of germination-aid in selection and evaluation for seedling emergence and vigor. Crop Science, v.2, n.1, p.176-7, 1962.

MARTINS, J.R. Aspectos da germinação de sementes e influência da luz no desenvolvimento, anatomia e composição química do óleo essencial em Ocimum gratissimum L. 2006. 187P. Dissertação (Mestrado - Área de Concentração em Fisiologia Vegetal) -
Departamento de Biologia, Universidade Federal de Lavras, Lavras.

MARTINS, J.R. et al. Influência da luz, temperatura e ácido giberélico na germinação de sementes de Ocimum gratissimum L. (Lamiaceae) e avaliação da qualidade fisiológica pelo teste de raios-X. Revista Brasileira de Plantas Medicinais, v.10, n.2, p.44-9, 2008a.

MARTINS, J.R. et al. Avaliação do crescimento e do teor de óleo essencial em plantas de Ocimum gratissimum L. cultivadas sob malhas coloridas. Revista Brasileira de Plantas Medicinais, v.10, n.4, p.102-7, 2008b.

MATASYOH, L.G. et al. Chemical composition and antimicrobial activity of the essential oil of Ocimum gratissimum L. growing in Eastern Kenya. African Journal of Biotechnology, v. 6, n. 6, p. 760-765, 2007.

NAGEL, M.; BÖRNER, A. The longevity of crop seeds stored under ambient conditions. Seed Science Research, v.20, n.1, p.1-12, 2010.

OGENDO, J. O. et al. Bioactivity of Ocimum gratissimum L. oil and two of its constituents against five insect pests attacking stored food products. Journal of Stored Products Research, v. 44, p. 328-334, 2008.

ROBERTS, E.H. Predicting the storage life of seeds. Seed Science and Technology, Zürich, v.1, n.4, 1973. p. 499-514.

SCALON, S.D.P.Q. et al. Armazenamento e tratamentos pré-germinativos em sementes de jacarandá (Jacaranda cuspidifolia mart.). Revista Árvore, v.30, n.2, p.179-85, 2006.

TRESENA, N. L.; MATA, M. E. R. C.; DUARTE, M. E. M.; MORAES, A. M.; DIAS, V. S. Qualidade fisiológica da semente de ipê rosa (Tabebuia hepptahylla (Vellozo) Toledo) submetidas à crioconservação. Revista Brasileira de Produtos Agroindustriais, Campina Grande, v. 11, p. 87-92, 2009

UEDA-NAKAMURA, T. et al. Antileishmanial activity of Eugenil-rich essential oil from Ocimum gratissimum. Parasitology International, v.55, n.2, p.99-105, 2006. 